\title{
Chestnut Flowers as Functionalizing Agents to Enhance the Antioxidant Properties of Highly Appreciated Traditional Pastry
}

\author{
Márcio Carocho, ${ }^{\mathrm{a}, \mathrm{b}}$, João C.M. Barreira ${ }^{\mathrm{a}, \mathrm{c}}$, Albino Bento ${ }^{\mathrm{a}}$, Patricia Morales ${ }^{\mathrm{b}}$, \\ Isabel C.F.R. Ferreira, ${ }^{a}$,
}

${ }^{a}$ Mountain Research Center (CIMO), ESA, Polytechnic Institute of Bragança, Portugal

${ }^{b}$ Department of Bromatology II, Faculty of Pharmacy, Complutense University of Madrid, Spain

${ }^{c}$ REQUIMTE/Department of Chemical Sciences, Faculty of Pharmacy, University of Oporto, Portugal

* Author to whom correspondence should be addressed (Isabel C.F.R. Ferreira; e-mail: iferreira@ipb.pt; telephone +351-273-303219) 


\begin{abstract}
Some studies have proven the antioxidant and antimicrobial potency of chestnut flowers both in the raw matrix and after extraction, and the consumption of their decoctions has been related to beneficial effects towards health. In recent years, due to controversy and ambiguous legislation of chemical conservatives, plant extracts have been successfully used as functionalizing agents in different matrixes, by displaying various beneficial effects towards the foodstuff and/or the consumer. In this manuscript, decoctions of chestnuts flowers as well as the dried flower were added to Portuguese traditional cakes that were then stored for 15 and 30 days, after which they were analysed for their antioxidant potential. The results were analysed by means of a 2 way ANOVA and a Linear Discriminant Analysis, concluding that storage time had a slightly higher influence in alteration of the antioxidant activity. DPPH and TBARS were the most improved parameters, regardless of the concentration added.
\end{abstract}

Keywords: Functionalized pastry; Chestnut Flowers; Antioxidant potential 


\section{Introduction}

Chestnuts are trees with an important impact on the Portuguese economy, mainly in the north-eastern part of the country, where the most of a total revenue of 32 million euros is produced in chestnut exportation ${ }^{1}$. Chestnut flowers (catkins), are by-products of the nut harvest, with no use after being fertilized and the development of the burr is started. Still, some of the ancient claims ${ }^{2}$ of the benefits of the consumption of infusions and decoctions of these flowers have been recently related to their antioxidant, antimicrobial and antitumor effects ${ }^{3,4}$.

The antioxidant activity is quite impressive, with $\mathrm{EC}_{50}$ (sample concentration providing $50 \%$ of effectiveness) values for the 2,2-diphenyl-1-picrylhydrazil (DPPH) assay being as low as $99 \mu \mathrm{g} / \mathrm{mL}$, and $15.24 \mu \mathrm{g} / \mathrm{mL}$ for the thiobarbituric reactive acid substances (TBARS) assay in decoctions and infusions, respectively. The high antimicrobial effects of these flowers could be related to their most representative polyphenol, trigalloylHHDP-glucoside followed by pedunculagin isomer, among other tannins ${ }^{4}$. The antioxidant activity present in edible natural matrices is quite important, not only to act as food preservers but also to mitigate reactive oxygen or nitrogen species that are produced in the normal metabolism of the human body, posing a threat to surrounding tissues. These species, also known as free radicals for not having stable orbitals, react vigorously with molecules in their vicinity and therefore causing damage which may lead to the development of many diseases, namely cancer, Alzheimer, gastric ulcers, diabetes, among others. Compounds like polyphenols, vitamins, minerals, and carotenoids, mainly found in plants and other natural matrices, have the ability to either mitigate these radicals or help regenerate antioxidants produced by the body, therefore being very appreciated as preventive factor for the mentioned diseases ${ }^{5,6}$. 
In the fast paced and global economy, the production, transportation and maintenance of sanity in the food chain has become under tight control, especially in developed countries. The maintenance of stability and sanity in food usually depends on chemical food additives, which delay contamination and halt deterioration. Although quite important for the food chain, these chemicals are becoming increasingly untrusted by many consumers throughout the world due to some known negative effects towards health that are attributed to them. Therefore, the use of natural additives, from plants and other matrices, has become more notorious, especially due to the added value of antioxidant benefits to the consumers ${ }^{7-9}$. Minced meat, meat balls, cheese and biscuits are some examples of food products functionalized with natural extracts ${ }^{10-13}$.

The cakes studied herein (known in Portugal as económicos) are made from flour, orange juice and cinnamon, being widely consumed for their good taste, although they don't have any extraordinary beneficial effect on health. In this manuscript, these traditional cakes were functionalized with dried chestnut flowers or their decoctions, and then kept for different storage periods. Finally, the final products were submitted to an extensive evaluation of their antioxidant properties to determine the best functionalizing ingredient (dried flowers or their decoctions), as well as to understand the antioxidant activity variation since the day of manufacture, and after 15 and 30 days of storage. The cakes have a validity date of one month, therefore it was used as the maximum storage time and the final analysis was carried out at that time, along with another one after 15 days. 


\section{Experimental}

\section{Standards and Reagents}

2,2-Diphenyl-1-picrylhydrazyl (DPPH), $\beta$-carotene, ascorbic acid, iron chloride, and potassium ferricyanide were obtained from Alfa Aesar (Ward Hill, MA, USA). FolinCiocalteu's reagent, iron sulfate, phosphate buffer, sodium carbonate, thiobarbituric acid, tricholoroacetic acid and Tween 80 were acquired from Fisher Scientific (Waltham, MA, USA). All other material and solutions were obtained by scientific retailers. All the water used in the methodology was treated with a purification system (TGI Pure Water Systems, Greenville, SC, USA).

\section{Flowers collection and preparation of decoctions}

Castanea sativa Mill. flowers of the cultivar Judia were collected in June 2013 in

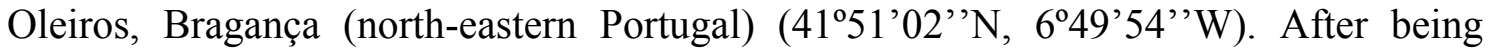
lyophilized (FreeZone 4.5, Labconco, Kansas, USA), they were milled down to a fine powder, and finally stored at $-5^{\circ} \mathrm{C}$ for further analysis. The decoctions were prepared following the standard procedure, used and characterized previously by the authors ${ }^{3}$, by adding the sample to cold water and letting it boil for 5 minutes and finally standing for another 5 minutes after turning the heat off.

\section{Preparation of the pastry}

For the preparation of the cakes the a traditional recipe was followed: 6 eggs were thoroughly mixed with $500 \mathrm{~g}$ of sugar, $1,05 \mathrm{~kg}$ of flour, $45 \mathrm{~g}$ of margarine and $30 \mathrm{~g}$ of warm olive oil. Then, $230 \mathrm{~mL}$ of pure orange juice, $35 \mathrm{~g}$ of orange peels, $350 \mathrm{~mL}$ of milk, $45 \mathrm{~mL}$ of firewater and $25 \mathrm{~g}$ of cinnamon were sequentially added to the mixture 
and mixed exhaustively. When all the ingredients were completely mixed and the dough reached a homogenous consistency, it was divided into 5 portions of $500 \mathrm{~g}$ each.

\section{Dried flowers and decoctions incorporation}

One portion was not incorporated, being used as the control sample; two samples were incorporated with two different quantities of the extract obtained from decoctions of chestnut flowers and the other two with different quantities of the dried flowers. For the decoctions, the best DPPH EC $\mathrm{E}_{50}$ value reported previously ${ }^{3}$ was used $(0.090 \mathrm{mg} / \mathrm{mL})$. For the $500 \mathrm{~g}$ of dough, adding the extract at the above concentration, $50 \mathrm{mg}$ were necessary, and for the second portion, a 2 fold quantity was added $(100 \mathrm{mg})$. For the dried flowers, the decoction extraction yield of $1 \mathrm{~g}$ was calculated (20\%), leading to an incorporation of $200 \mathrm{mg}$ for one portion of the dough, and once again, the double amount (400 mg) for the other portion.

The samples were named "C" for control, "D50" and "D100" for the portions containing 50 and $100 \mathrm{mg}$ of decoction, respectively, and "F200" and "F400" for the portions incorporated with the dried flowers. After the incorporation off all the portions, they were divided into individual cakes and finally baked in an oven at $170{ }^{\circ} \mathrm{C}$ for 15 minutes.

\section{Storage}

After baking, the five different samples of cakes were left do cool down for a few minutes and after collection of a representative number of cakes of each for immediate analysis, they were placed in sealed plastic bags and stored at room temperature $\left(18 \sim 23^{\circ} \mathrm{C}\right)$ away from light for 15 and 30 days, respectively. 


\section{Evaluation of antioxidant activity}

The in vitro antioxidant activity assays were performed following the previously described methodology by Barros, Oliveira, Carvalho, \& Ferreira ${ }^{14}$. The cakes were frozen, lyophilized, milled down and extracted twice with water, then re-dissolved to a known concentration of $100 \mathrm{mg} / \mathrm{mL}$. This solution was further diluted to different concentrations to be used in the antioxidant activity assays. DPPH radical-scavenging activity was evaluated using an ELX800 microplate Reader (Bio-Tek Instruments, Inc.; Winooski, VT, USA) and calculated as a percentage of DPPH discolouration after 1 hour of incubation with the antioxidant extract, using the formula: $\left[\left(A_{\mathrm{DPPH}}-A_{S}\right) / A_{\mathrm{DPPH}}\right]$ $\times 100$, where $A_{S}$ is the absorbance of the solution containing the sample at $515 \mathrm{~nm}$, and $A_{\mathrm{DPPH}}$ is the absorbance of the DPPH solution. The reducing power was evaluated by the capacity to reduce $\mathrm{Fe}^{3+}$ to $\mathrm{Fe}^{2+}$, measuring the absorbance at $690 \mathrm{~nm}$ in the microplate reader mentioned above. Inhibition of $\beta$-carotene bleaching was evaluated through the $\beta$-carotene/linoleate assay; the neutralization of linoleate free radicals avoids $\beta$-carotene bleaching, which is measured by the formula: ( $\beta$-carotene absorbance after $2 \mathrm{~h}$ of assay/initial absorbance $) \times 100$. Lipid peroxidation inhibition in porcine $(S u s$ scrofa) brain homogenates was evaluated by the decrease in thiobarbituric acid reactive substances (TBARS); the colour intensity of the malondialdehyde-thiobarbituric acid (MDA-TBA) was measured by its absorbance at $532 \mathrm{~nm}$; the inhibition ratio (\%) was calculated using the following formula: $[(A-B) / A] \times 100 \%$, where $A$ and $B$ were the absorbance of the control and the sample solution, respectively. The results of the antioxidant activity were expressed as $\mathrm{EC}_{50}$ values. Total phenolics were determined by the Folin-Ciocalteu assay, measuring the absorbance at $765 \mathrm{~nm}$. Gallic acid was used as a standard, and the results were expressed as mg of gallic acid equivalents (GAE) per $g$ of extract. 


\section{Statistical Analysis}

In order to have representative results, a pool of nine cakes was used for each case (control, cakes incorporated with $C$. sativa flower decoctions at $\mathrm{EC}_{50}$ or $2 \times \mathrm{EC}_{50}$ concentrations and cakes supplemented with $C$. sativa dried flowers at $\mathrm{EC}_{50}$ or $2 \times \mathrm{EC}_{50}$ concentrations), comprising a total of 45 cakes. From each pool, three individual samples were used and all the assays were carried out in triplicate. Data was expressed as means \pm standard deviations, maintaining the decimal places allowed by the magnitude of standard deviation.

An analysis of variance (ANOVA) with type III sums of squares was performed using the GLM (General Linear Model) procedure of the SPSS software. The dependent variables were analyzed using 2-way ANOVA, with the factors "storage time" (ST) and "concentration" $(\mathrm{C})$. In this case, when a statistically significant interaction $(\mathrm{ST} \times \mathrm{C})$ was detected, the two factors are evaluated simultaneously by the estimated marginal means plots for the two levels of each factor. Alternatively, if no statistical significant interaction is verified, means are compared using suitable multiple comparison tests. The equality of variances was verified through a Levene's test.

In addition, linear discriminant analysis (LDA) was used to compare the effect of the ST and $\mathrm{C}$ on the antioxidant activity of functionalized cakes. A stepwise technique, using the Wilks' $\lambda$ method with the usual probabilities of $F$ (3.84 to enter and 2.71 to remove), was applied for variable selection. This procedure uses a combination of forward selection and backward elimination processes, where the inclusion of a new variable is preceded by verifying the significance of all variables previously selected. The basic purpose of a discriminant analysis is estimating the relationship between a single categorical dependent variable (the cake formulation, in this case) and a set of quantitative independent variables (the $\mathrm{EC}_{50}$ values obtained in all antioxidant assays). 
With this approach, it is possible to determine which of the independent variables account most for the differences in the average score profiles of the different cakes. To verify the significance of canonical discriminant functions, the Wilks' $\lambda$ test was applied. A leaving-one-out cross-validation procedure was carried out to assess the model performance. The graph representations were included to assess the distinctiveness of the analyzed cakes based on their antioxidant activity. All statistical tests were performed at a $5 \%$ significance level $^{15}$.

\section{Results and Discussion}

The high antioxidant potential of chestnut flowers was previously reported, emphasizing the $\mathrm{EC}_{50}$ values of their methanolic (DPPH: $70 \mu \mathrm{g} / \mathrm{mL}$, reducing power: $70 \mu \mathrm{g} / \mathrm{mL}, \beta$ carotene bleaching: $110 \mu \mathrm{g} / \mathrm{mL}$ and TBARS formation inhibition: $30 \mu \mathrm{g} / \mathrm{mL})^{14}$, and aqueous (DPPH: $75 \mu \mathrm{g} / \mathrm{mL}$, reducing power: $87 \mu \mathrm{g} / \mathrm{mL}, \beta$-carotene bleaching: 161 $\mu \mathrm{g} / \mathrm{mL}$ and TBARS formation inhibition: $10 \mu \mathrm{g} / \mathrm{mL})^{3,16}$ extracts. These rarely low $\mathrm{EC}_{50}$ values justify choosing $C$. sativa flowers to functionalize foods. Regarding the solvent type, due to the known toxicity of methanol, opting for aqueous extracts was a better choice, among which decoctions were reported as containing the highest amounts in phenolic compounds ${ }^{3}$.

The $\mathrm{EC}_{50}$ values obtained for each antioxidant assay are presented (Table 1) as the mean value of each storage time (ST), including the different concentrations used to functionalize the cakes, and also the mean value of each concentration (C), including the results for all ST. This approach allows electing the best condition for each factor, independently of the effect caused by the other analyzed factor. According to this approach, the tabled standard deviations may not be simply seen as a measure of the accuracy of applied methodologies, since they reflect also variations of the non-fixed 
factor (ST or C). In order to ease the interpretation of results, Table 1 was divided in samples prepared with decocted extracts or by the direct addition of dried flowers. In all cases, $\mathrm{ST} \times \mathrm{C}$ interaction was a significant $(p<0.001)$ source of variation. Accordingly, and despite presenting the least squares means for both effects, no multiple comparisons could be performed. Nevertheless, from the analysis of the EMM (estimated marginal means) plots (data not shown) some overall conclusions can be outlined.

The effects of $C$. sativa flower (either directly added or previously decocted) on the antioxidant activity of these traditional cakes were found to be statistically significant ( $p$ $<0.001)$. The storage time was also determinant, proving to have a strong interaction with each of the functionalizing agents (dried flowers and decoctions), besides exerting a significant effect per se. Nevertheless, and most likely because the applied antioxidant assays are based in different reaction mechanisms, the effects observed for each assay did not show the same behavior.

Regarding the functionalization with flower decoctions, the power to scavenge DPPH was improved in the functionalized cakes (independently of the added concentration), especially after a 15 day period. The reducing power, however, did not show a good response in cakes functionalized with a $2 \times \mathrm{EC}_{50}$ concentration, and none of the assayed periods exerted significant differences in the obtained results. On the other hand, the $2 \times \mathrm{EC}_{50}$ concentration gave the best inhibitory activity against $\beta$-carotene bleaching, despite this effect was somehow lost after 30 days of storage (this decrease was observed independently of the used concentration). The inhibition of TBARS formation was significantly increased in the functionalized samples, independently of using a concentration corresponding to the $\mathrm{EC}_{50}$ or its double, which in both cases showed the best results after 30 days of storage. This result was in agreement with the observed for phenolic content, which tended to be higher after 30 days of storage. 
Concerning samples functionalized by direct addition of dried $C$. sativa flowers, the effects on DPPH scavenging activity and $\beta$-carotene bleaching inhibition were similar to those obtained with flower decoctions. However, the effect on reducing power was different, with an increased activity measured in samples supplemented with a $2 \times \mathrm{EC}_{50}$ concentration powder, and a worst scenario in samples stored during 15 days. Also, the best inhibitory effect over TBARS formation inhibition was verified in samples not submitted to storage. In line with the result obtained in cakes functionalized with decoctions, the content in phenolic compounds was also higher in stored samples.

The differences observed among cakes functionalized with decocted extracts or dried flowers indicate that the chemical composition of each ingredient is necessarily different. In addition, the $\mathrm{EC}_{50}$ values obtained when using samples functionalized with dried flowers were slightly lower, when compared to those obtained using decoctions as functionalizing agents. Nevertheless, the functionalized cakes showed higher antioxidant activity and phenolic contents when compared to the control. Similar results were previously published, using different botanical sources, namely mango peel and Moringa oleifera extracts in biscuits ${ }^{12,17,18}$.

The phenolic composition of $C$. sativa flower decoctions is known to include flavonols (quercetin, isorhamnetin, kaempferol and myricetin derivatives), hydrolysable tannins (galloyl and hexahydroxydiphenol derivatives) and flavanols ((+)-catechin $)^{3}$, compounds that might explain the antioxidant activity observed in the final products. In Figure 1, it might be seen that, neither the exterior, nor the interior appearance, were changed by adding dried flowers (the decoctions were lighter than flowers). In fact, the amounts used to functionalize these cakes were very low, which explains the inexistence of higher differences in antioxidant activity or phenolic contents in functionalized samples. 
In order to understand the true effect of using flower decoctions or dried flowers, a multivariate discriminant analysis was applied, considering the results for all the variables. Following the same reasoning as in Table 1, the analyses were separated according to the type of functionalizing agent. The discriminant ability of the antioxidant activity results can be assessed from the obtained classification performance, given by the percentage of correctly classified groups.

Regarding samples functionalized with flower decoctions, the ST exerted higher influence, since $100.0 \%$ of the samples were correctly classified, both for the original groups and for the cross-validation. The classification ability was lower for $\mathrm{C}$ effect, resulting in $85.2 \%$ of accuracy for the original groups and for the cross-validation, as deduced from the leaving-one-out cross-validation procedure. In both cases, two significant ( $p<0.001$ for the Wilks' $\lambda$ test) discriminant functions, including $100.0 \%$ of the variance of the experimental data (Figure 2).

Table 2 shows the eigenvalues and the correlations of the discriminating functions with each variable. According to the magnitude of the different correlations, it might be concluded that the effect of ST was mostly reflected in DPPH scavenging activity (function 1) and phenolic content (function 2). A similar result was obtained in what concerns the effect of $\mathrm{C}$.

For the samples functionalized with dried flower, the ST exerted again higher influence, since $100.0 \%$ of the samples were correctly classified, both for the original groups and for the cross-validation. The classification ability was slightly lower for $\mathrm{C}$ effect, resulting in $96.3 \%$ of accuracy for the original groups and $93.8 \%$ for the crossvalidation, as deduced from the leaving-one-out cross-validation procedure. In both cases, two significant discriminant functions were also defined, including $100.0 \%$ of the variance of the experimental data (Figure 3). 
As it can be depicted from Table 2 that reducing power was the variable most correlated to function 1 and DPPH scavenging activity to function 2 for the ST effect, while DPPH scavenging activity (function 1) and phenolic content (function 2) were the most discriminant variables regarding the effect of $\mathrm{C}$.

\section{Conclusions}

Overall, the functionalized cakes showed significant differences, independently of the storage time, despite no general conclusion could be drawn regarding the use of $\mathrm{EC}_{50}$ or $2 \times \mathrm{EC}_{50}$ concentrations to enhance the antioxidant activity: DPPH scavenging activity was similar for both concentrations using flower decoctions or dried flowers; reducing power was better using $\mathrm{EC}_{50}$ of decoction and $2 \times \mathrm{EC}_{50}$ of dried flowers; $\beta$-Carotene bleaching inhibition was enhanced with $2 \times \mathrm{EC}_{50}$, using decoctions or dried flowers; TBARS formations inhibition was similar for both concentrations and both functionalizing agents. The phenolic content was higher after 30 days of storage, especially in functionalized samples.

In conclusion, the functionalized cakes showed increased antioxidant activity and phenolic content, without causing visible changes in the inner and outer appearance of the final product. The obtained results were also useful to define the most adequate concentration, functionalizing agent (decoction or dried flower) and the most suitable storage time ( 0,15 or 30 days), as described above.

\section{Competing interests}

The authors declare no competing financial interest. 


\section{Acknowledgments}

The authors are grateful to M. Ferreira e Filhas Lda. (Pão de Gimonde) for the cakes recipe, and to the Foundation for Science and Technology (FCT, Portugal), for financial support to the CIMO research center (PEst-OE/AGR/UI0690/2011) and for J.C.M. Barreira's Post-Doctoral grant (BPD/72802/2010).

\section{References}

1 INE, Instituto Nacional de Estatística, Portugal. Chestnuts national statistics, 2012, http://www.ine.pt/xportal/xmain?xpid=INE\&xpgid=inepublicacoes\&PUBLICACOE Spub boui $=153380933 \&$ PUBLICACOESmodo=2 (accessed June 2014).

2 J.M. Neves, C. Matos, C. Moutinho, G. Queiroz, L.R. Gomes, J. Ethnopharmacol., $2009,124,70-283$.

3 M. Carocho, L. Barros, A. Bento, C. Santos-Buelga, P. Morales, I.C.F.R. Ferreira, BioMed Res. Int., 2014, Article ID 232956, 7 pages.

4 M. Carocho, R.C. Calhelha, M.J.R.P. Queiroz, A. Bento, P. Morales, M. Soković, I.C.F.R. Ferreira, Ind. Crops. Prod., 2014, submitted.

5 D.V. Ratnam, D.D. Ankola, V. Bhardwaj, D.K. Sahana, M.N.V.R. Kumar, J. Control. Rel., 2006, 113, 189-207.

6 M. Carocho, I.C.F.R Ferreira, Food Chem. Toxicol., 2013, 51, 15-25.

7 L. Day, R.B. Seymour, K.F. Pitts, I. Konczak, L. Lundin, Trend Food Sci. Technol., 2009, 20, 388-395.

8 M.S. Brewer, Comp. Rev. Food Sci. Food Safety, 2011, 10, 221-247.

9 M. Carocho, M.F. Barreiro, P. Morales, I.C.F.R. Ferreira, Comp. Rev. Food Sci. Food Safety, 2014 (in press). 
10 J. Fernández-López, N. Zhi, L. Aleson-Carbonell, J.A. Pérez-Alvarez, V. Kuri, Meat Sci., 2005, 69, 371-380.

11 M. Østerile, J. Lerfall, Food Res. Int., 2005, 38, 925-929.

12 V. Reddy, A. Urooj, A. Kumar, Food Chem., 2005, 90, 317-321.

13 B. Shan, Y. Cai, J.D. Brooks, H. Corke, J. Med. Food, 2011, 14, 284-290.

14 L. Barros, S. Oliveira, A.M. Carvalho, I.C.F.R. Ferreira, Ind. Crops Prod, 2010, 32, $572-579$.

15 A. López, P. García, A. Garrido, Food Chem., 2008, 106, 369-378.

16 J.C.M. Barreira, I.C.F.R. Ferreira, M.B.P.P. Oliveira, J.A. Pereira, Food Chem., 2008, 107, 1106-1113.

17 C.M. Ajila, K. Leelavathi, U.J.S.P. Rao, J. Cereal Sci., 2008, 48, 319-326.

18 F. Zucco, Y. Borsuk, S.D. Arntfield, LWT-Food Sci. Technol., 2011, 44, 2070-2076. 
Table 1. Antioxidant properties obtained for the extracts of chestnut cultivars (CC). The results are presented as mean $\pm \mathrm{SD}$. Values are presented as $\mathrm{EC}_{50}$ values $(\mathrm{mg} / \mathrm{mL})$ for all assays except phenolic content, expressed as mg GAE/g extract.

DPPH scavenging Reducing TBARS formation $\beta$-Carotene Phenolic
activity power inhibition bleaching inhibition content

\begin{tabular}{|c|c|c|c|c|c|c|}
\hline & & & er decoct & & & \\
\hline & 0 days & $171 \pm 43$ & $8.2 \pm 0.5$ & $3 \pm 1$ & $8.2 \pm 0.5$ & $3.7 \pm 0.4$ \\
\hline CT & 15 days & $122 \pm 50$ & $6 \pm 2$ & $8 \pm 5$ & $9 \pm 2$ & $2.6 \pm 0.5$ \\
\hline & 30 days & $229 \pm 12$ & $5.1 \pm 0.2$ & $2 \pm 1$ & $14 \pm 7$ & $5 \pm 1$ \\
\hline & $p$-value $(\mathrm{n}=27)$ & $<0.001$ & $<0.001$ & $<0.001$ & $<0.001$ & $<0.001$ \\
\hline & $0 \mathrm{mg} / \mathrm{mL}$ & $215 \pm 21$ & $6 \pm 2$ & $6 \pm 6$ & $9 \pm 2$ & $4 \pm 1$ \\
\hline & $\mathrm{EC}_{50}$ & $151 \pm 65$ & $6 \pm 1$ & $3 \pm 1$ & $14 \pm 7$ & $4 \pm 1$ \\
\hline & $2 \times \mathrm{EC}_{50}$ & $155 \pm 54$ & $8 \pm 2$ & $4 \pm 1$ & $7 \pm 2$ & $4 \pm 2$ \\
\hline & $p$-value $(\mathrm{n}=27)$ & $<0.001$ & $<0.001$ & $<0.001$ & $<0.001$ & $<0.001$ \\
\hline$\overline{\mathrm{STX}}$ & $p$-value $(\mathrm{n}=81)$ & $<0.001$ & $<0.001$ & $<0.001$ & $<0.001$ & $<0.001$ \\
\hline & & & ed flowe & & & \\
\hline & 0 days & $88 \pm 99$ & $7 \pm 2$ & $1.5 \pm 0.5$ & $7 \pm 2$ & $2.9 \pm 0.4$ \\
\hline CT & 15 days & $128 \pm 45$ & $3.9 \pm 0.5$ & $7 \pm 5$ & $10 \pm 2$ & $5 \pm 1$ \\
\hline & 30 days & $217 \pm 12$ & $7 \pm 2$ & $3 \pm 2$ & $9 \pm 2$ & $5 \pm 1$ \\
\hline & $p$-value $(\mathrm{n}=27)$ & $<0.001$ & $<0.001$ & $<0.001$ & $<0.001$ & $<0.001$ \\
\hline & $0 \mathrm{mg} / \mathrm{mL}$ & $215 \pm 21$ & $6 \pm 2$ & $6 \pm 6$ & $9 \pm 2$ & $4 \pm 1$ \\
\hline C & $\overline{\mathrm{EC}_{50}}$ & $109 \pm 80$ & $7 \pm 2$ & $3 \pm 2$ & $10 \pm 2$ & $4 \pm 2$ \\
\hline 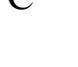 & $2 \times \mathrm{EC}_{50}$ & $109 \pm 80$ & $4 \pm 1$ & $3 \pm 2$ & $7 \pm 3$ & $5 \pm 1$ \\
\hline & $p$-value $(\mathrm{n}=27)$ & $<0.001$ & $<0.001$ & $<0.001$ & $<0.001$ & $<0.001$ \\
\hline ST $x$ & -value $(\mathrm{n}=81)$ & $<0.001$ & $<0.001$ & $<0.001$ & $<0.001$ & $<0.001$ \\
\hline
\end{tabular}

Data was expressed as means \pm standard deviations, maintaining the decimal places allowed by the magnitude of standard deviation. 
Table 2. Eigenvalues, percentage of variance and standardized coefficients for the obtained linear discriminant functions.

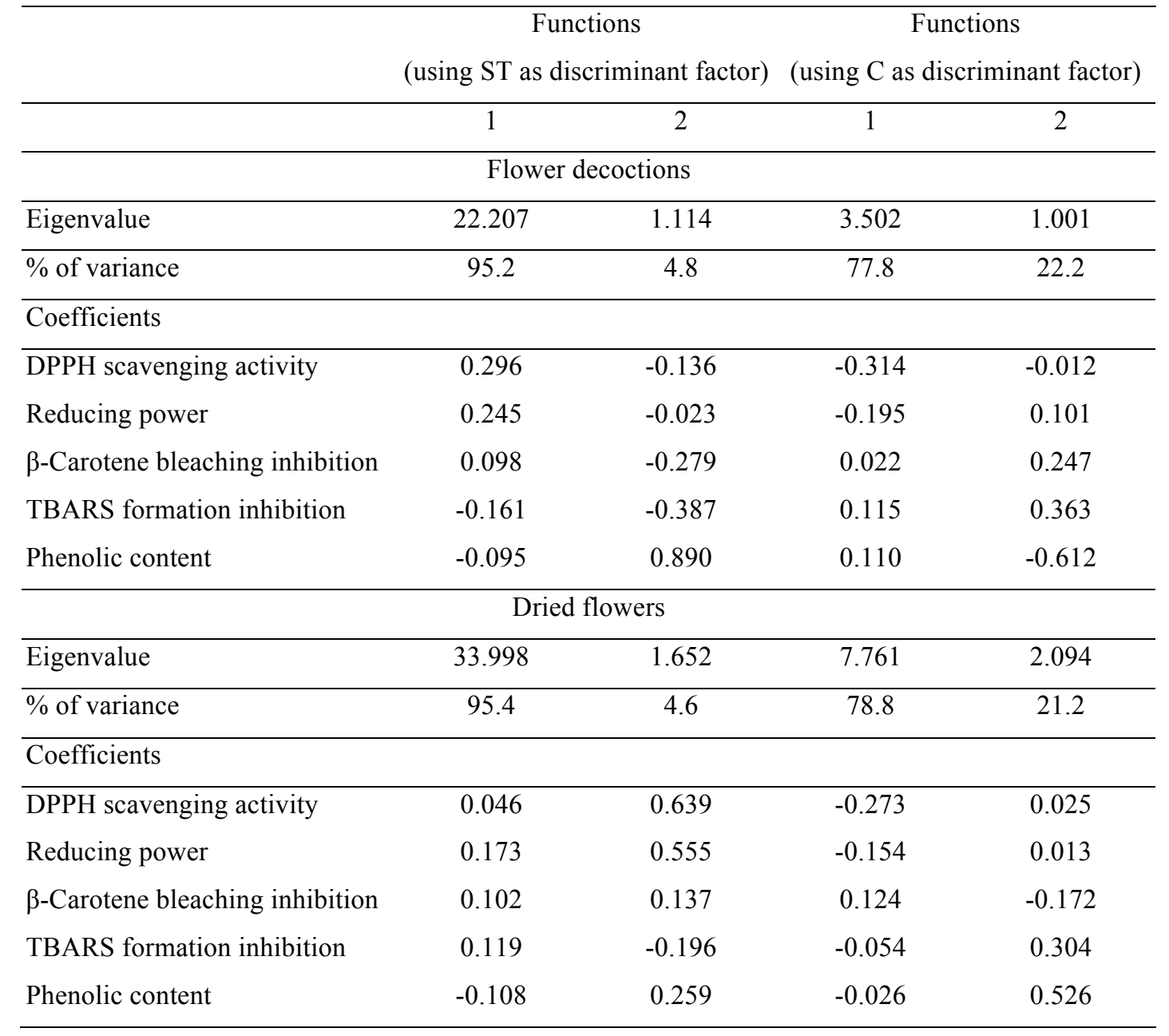


Figure 1. Cakes functionalized with $C$. sativa dried flowers. A- control; Bfunctionalized with amounts corresponding to $\mathrm{EC}_{50}$; $\mathrm{C}$ - functionalized with amounts corresponding to $2 \times \mathrm{EC}_{50}$. 

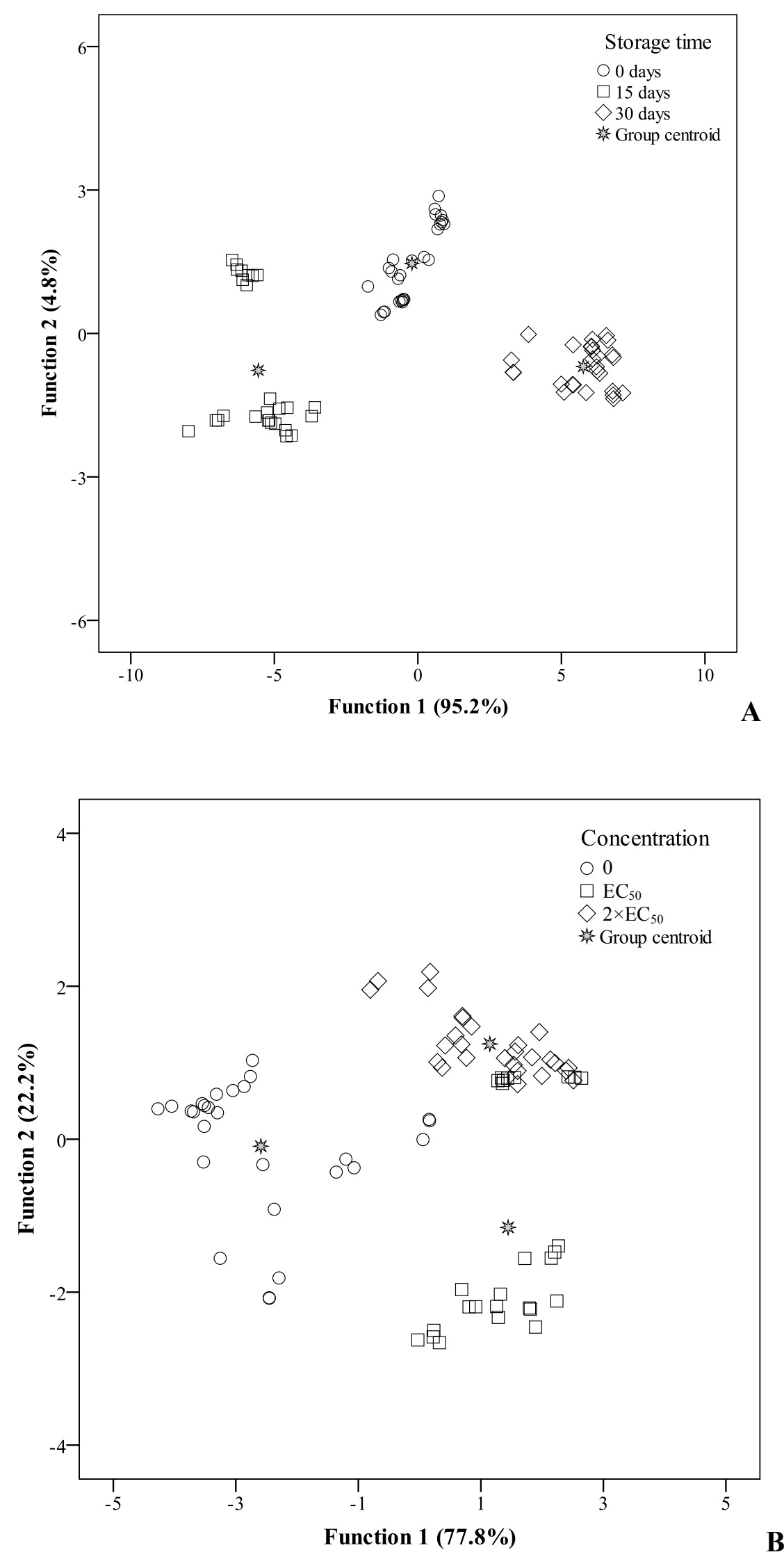
Figure 2. Discriminant scores scatter plot of the canonical functions defined for antioxidant activity results according with storage time (A) and concentration (B) for cakes functionalized with $C$. sativa decoctions. 

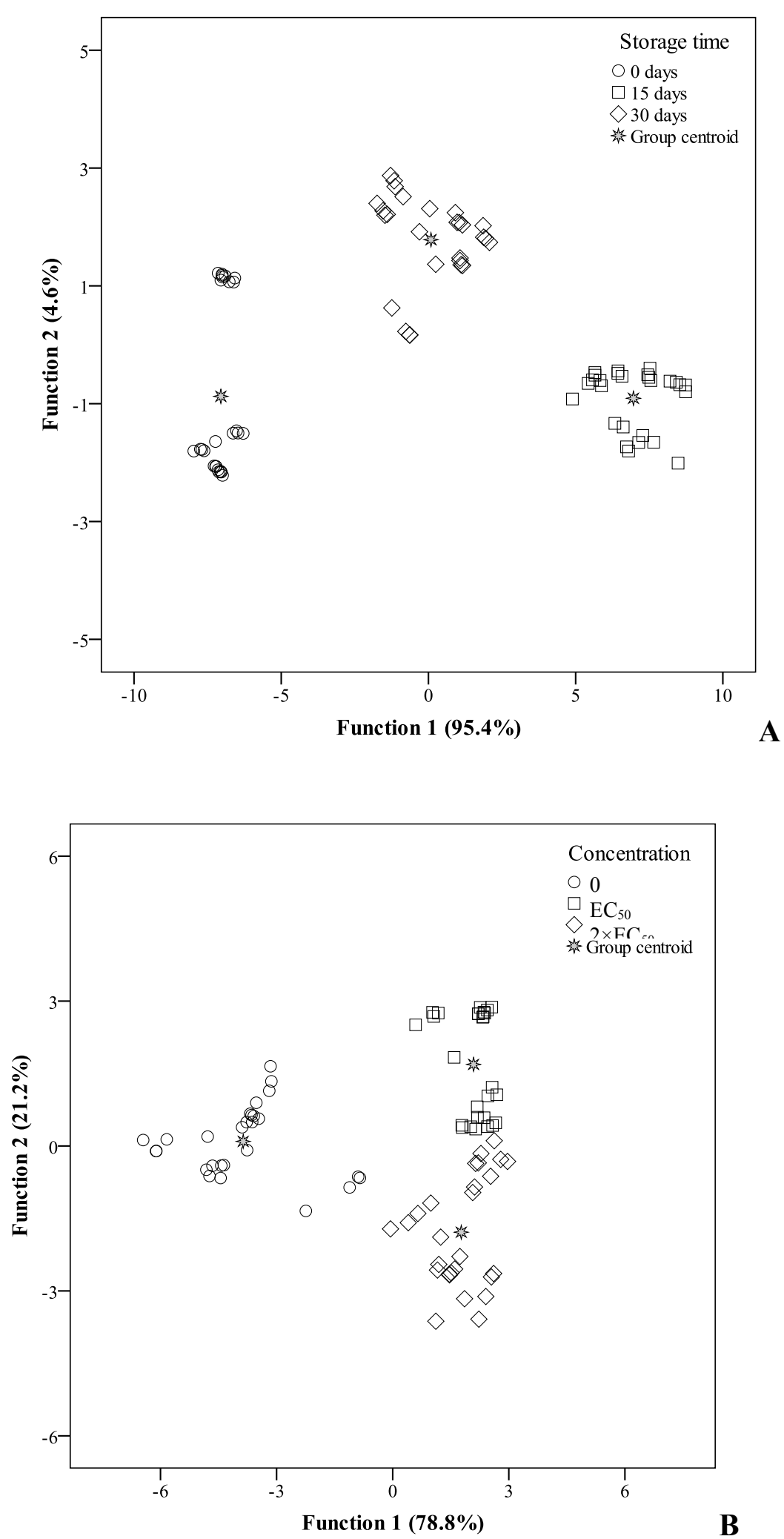

Figure 3. Discriminant scores scatter plot of the canonical functions defined for antioxidant activity results according with storage time (A) and concentration (B) for cakes functionalized with $C$. sativa dried flowers. 